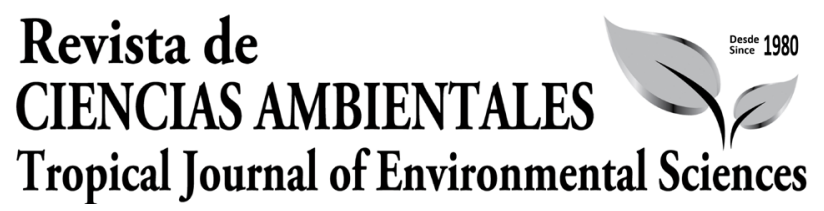

Revista de Ciencias Ambientales (Trop J Environ Sci) e-ISSN: 2215-3896 (Enero-Junio, 2020) . Vol 54(1): 190-199. DOI: https://doi.org/10.15359/rca.54-1.11

Open Access: www.revistas.una.ac.cr/ambientales e-mail: revista.ambientales@una.ac.cr Salazar $\mathrm{H}$.

\title{
BIOGRAFÍA
}

\section{Alberto Manuel Brenes: primer botánico costarricense}

\author{
Alberto Manuel Brenes: The First Costa Rican Botanist
}

Hámer Salazar ${ }^{1}$

\section{Introducción}

Alberto Manuel Brenes fue un destacado botánico de principios del siglo XX. Su impacto en el conocimiento de la diversidad de plantas en Costa Rica fue tal, que Juvenal Valerio Rodríguez, director del Museo Nacional, así como discípulo y colega suyo, lo llamó el botánico nacional (Valerio, 1940). Sin embargo, su carácter hosco y solitario, la renuencia a hablar de sí mismo y la ausencia de publicaciones propias, han permitido dibujar un halo de misterio sobre su figura. De hecho, en su natal San Ramón, incluso la gente más ilustre es poco lo que conoce acerca de él.

La primera reseña biográfica sobre Brenes la realizó Fulvia Monge de Zamora, siete años antes de la muerte del botánico (Monge, 1941). Asimismo, Vitalía Sáenz Bejarano, quien fue su asistente en el Museo Nacional, escribió una breve biografía de él, y se lamentaba de no haber podido obtener más información, pues Brenes quería permanecer en el anonimato, cuando le confesó: "Prefiero y deseo que se me permita gozar de este spleen en que vivo, y que no se diga nada que lo turbe, mientras yo viva” (Sáenz, 1945).

Incluso Trino Echavarría, quien fuera su amigo personal, también intentó recopilar información del botánico, pero cuando se la solicitaba "sonreía el viejito con un rictus amargo... y no daba nada en respuesta... luego encauzaba la conversación sobre las orquídeas, a esas flores de encanto y hablaba de ellas con entusiasmo de enamorado" (Echavarría, 1944). Finalmente, Olger Salas, representante de la Secretaría de Educación, decía de Brenes que "las fulguraciones de su inteligencia iluminaron la montaña y él solo penetró en el bosque para traernos a los hombres enseñanzas de incalculable valor" (Salas, 1949).

1 Biólogo. Profesor jubilado, Universidad de Costa Rica. ahamersalazar@gmail.com

\begin{tabular}{|c|c|c|}
\hline 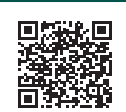 & (c) (i) (5) (2) & 190 \\
\hline
\end{tabular}




\section{Revista de CIENCIAS AMBIENTALES Tropical Journal of Environmental Sciences}

Revista de Ciencias Ambientales (Trop J Environ Sci) e-ISSN: 2215-3896 (Enero-Junio, 2020) . Vol 54(1): 190-199. DOI: https://doi.org/10.15359/rca.54-1.11

Open Access: www.revistas.una.ac.cr/ambientales e-mail: revista.ambientales@una.ac.cr

Salazar H.

Aunque reacio a los reconocimientos públicos, la Asociación de Cultura Ramonense tuvo el tino de rendirle un cálido homenaje en vida, cuatro años antes de su deceso, ocurrido el 18 de mayo de 1948. En efecto, en la significativa fecha del 15 de setiembre de 1944, día conmemorativo de la independencia de Centroamérica, los escolares desfilaron desde su casa (Figura 1) hasta el parque de la ciudad — que hoy porta su nombre-, donde se descubrió una placa dedicada a él; además, su retrato fue desvelado en el salón de actos de la Escuela Jorge Washington.

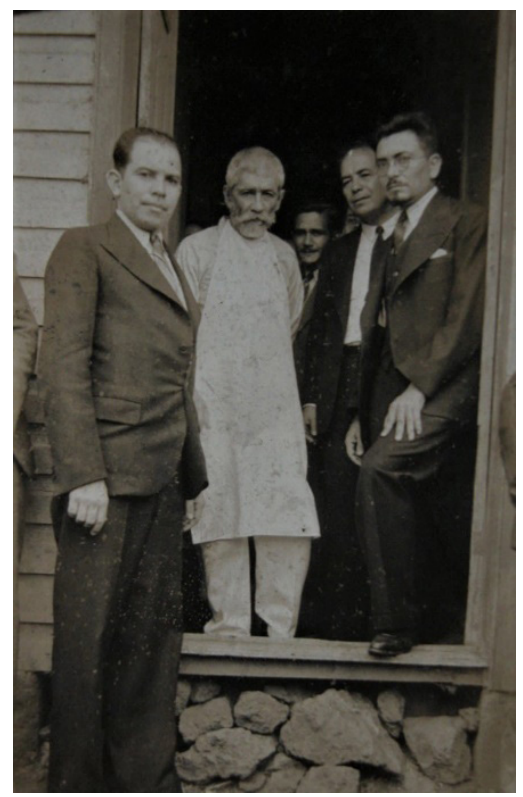

Figura 1. Alberto Brenes en su casa el día del homenaje. A mano derecha aparece Juvenal Valerio (con anteojos). Figure 1. Alberto Brenes at home on the day of the tribute. On the right hand appears Juvenal Valerio (with glasses).

Asimismo, por fortuna, y con gran justicia, pocos días antes de cumplirse el centenario de su nacimiento, el 11 de agosto de 1970 la Asamblea Legislativa lo declaró a Benemérito de la Ciencia (La Gaceta, 1970). Además, por iniciativa del diputado ramonense Deseado Barboza Ruiz, en 1974 se aprobó la Ley No. 5612, cuyo propósito era la emisión de una colección de sellos postales. Parte de los recursos que la venta de estas estampillas generaría, se utilizaría en la "edición de un libro alusivo a la vida y la obra del profesor Alberto Manuel Brenes Mora", pero este objetivo nunca se cumplió. No fue sino hasta la publicación de Salazar (2009), que se recopiló información suficiente sobre Brenes, obra que sirve de fuente para el presente artículo.

\section{Infancia y adolescencia}

El 2 de setiembre del año 1870 nació, en el distrito Central de la Villa de San Ramón, un niño que fue bautizado con el nombre de Manuel Alberto de Jesús, como hijo natural de Catalina

\begin{tabular}{|c|c|c|}
\hline 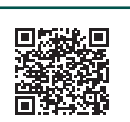 & (c) (1) (8)(2) & 191 \\
\hline
\end{tabular}




\section{Revista de CIENCIAS AMBIENTALES Tropical Journal of Environmental Sciences}

Revista de Ciencias Ambientales (Trop J Environ Sci) e-ISSN: 2215-3896 (Enero-Junio, 2020) . Vol 54(1): 190-199. DOI: https://doi.org/10.15359/rca.54-1.11

Open Access: www.revistas.una.ac.cr/ambientales e-mail: revista.ambientales@una.ac.cr

Salazar H.

Brenes Mora. De hogar humilde, debió trabajar siendo un niño de edad escolar para ayudarle a su madre en el sostenimiento suyo y el de sus hermanos menores (Monge, 1941).

Una vez que terminó sus estudios primarios, trabajó en varias boticas de San Ramón, donde recibió las primeras lecciones de botánica. Inició su educación secundaria en el Colegio Horacio Mann, de San Ramón. Gamboa y Sánchez (1970) describen al joven Brenes de inteligencia despejada y de criterio analítico, quien destacaba en forma sobresaliente entre sus compañeros, lo cual le favoreció para su traslado al Liceo de Costa Rica, donde concluyó la educación secundaria.

\section{De San Ramón a Europa}

En reconocimiento a su talento e interés por la botánica, y gracias a la participación directa de Bernardo Soto Alfaro, presidente de la República, se le concedió una beca para que cursara estudios superiores en Europa (Gamboa y Sánchez, 1970; Echavarría, 1944). Por tanto, en abril de 1890, se trasladó a Francia y se instaló en París, donde estuvo aprendiendo el idioma francés, y de allí pasó a Suiza, donde cursó su primer año de estudios en la entonces Academia de Lausanne. No satisfecho con los estudios que allí seguía, pidió su traslado a la Universidad de Ginebra, donde, sin interrupción, prosiguió sus estudios en la Facultad de Ciencias (Monge, 1941; Echavarría, 1944).

No hay evidencias de que el botánico ramonense lograra graduarse en la Universidad de Ginebra (Salazar, 2009). En este sentido, Jiménez (1945), uno de sus biógrafos, de manera implícita sugiere que don Alberto no obtuvo títulos, al asegurar que: "su vuelta al país, en 1898, con más sabiduría que diplomas, fue acogida con frialdad y tal vez con incomprensión por las instituciones oficiales" (Jiménez, 1945). Se supone que una de las razones por las cuales no culminó sus estudios, fue por el abandono que el gobierno de Rafael Iglesias Castro hizo de los estudiantes becados en el extranjero, y fue gracias al concurso del presbítero José Piñeiro, cura párroco de San Ramón, que se le envió el dinero suficiente para su regreso (Cruz, 1990).

\section{El botánico nacional}

Durante la administración liberal de Bernardo Soto, y siendo ministro de Educación el gran reformador Mauro Fernández Acuña, se crearon el Colegio Superior de Señoritas y el Liceo de Costa Rica. Fue durante este período cuando Fernández, con la firme convicción de brindar una educación de calidad en aquellas instituciones de educación, le solicitó a Manuel María Peralta, quien fungía como ministro plenipotenciario en Europa, que contratara profesores para completar la planilla del cuerpo docente, para aquellos privilegiados estudiantes. Como parte del grupo de profesores contratados, llegó el naturalista suizo Henri François Pittier.

Brenes fue contratado por Pittier para que hiciera diez colecciones de plantas de 500 especies cada una (Jiménez, 1945), lo que significó recolectar alrededor de 5000 especímenes. Hecho esto, Brenes cargó los paquetes en carretas tiradas por bueyes y se dirigió hasta la estación del

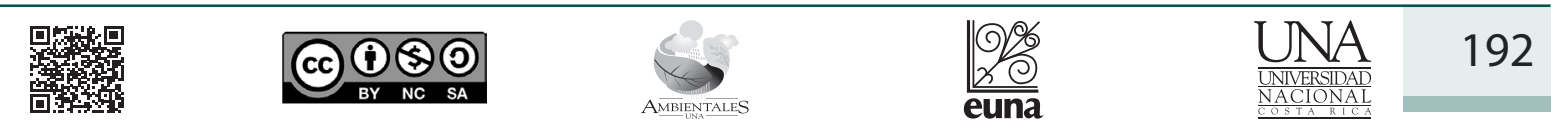




\section{Revista de CIENCIAS AMBIENTALES Tropical Journal of Environmental Sciences}

Revista de Ciencias Ambientales (Trop J Environ Sci) e-ISSN: 2215-3896 (Enero-Junio, 2020) . Vol 54(1): 190-199. DOI: https://doi.org/10.15359/rca.54-1.11

Open Access: www.revistas.una.ac.cr/ambientales e-mail: revista.ambientales@una.ac.cr

Salazar H.

Ferrocarril Eléctrico al Pacífico, en Río Grande de Atenas, para enviarlas hasta la capital (Jiménez, 1945).

Con las muestras en San José, se instaló en la torre del viejo edificio del Museo Nacional, trabajando de manera incansable día y noche, al punto de que incluso dormía ahí. Como parte del convenio con Pittier, debió montar cada uno de los especímenes botánicos en cartulinas de herbario, ordenarlas y etiquetarlas.

Fue en la administración de Julio Acosta García, coterráneo de Brenes, iniciada en 1920, que a Brenes se le nombró jefe de la Sección de Botánica del Museo Nacional, puesto que le permitió realizar una extraordinaria colección de plantas.

Sáenz (1945), hizo un recuento, ejemplar por ejemplar, de la colección de Brenes y contabilizó un total de 22638 especímenes, hasta 1942. Cooperó con científicos de la talla de Rudolf Schlechter (Herbario de Berlín), Georg Cufodontis (Museo de Historia Natural de Austria), Paul C. Standley (Museo de Chicago) y Oakes Ames (Universidad de Harvard), quien elaboró la lista de orquídeas para la flora de Costa Rica, publicada por Standley (1937). Por esta razón, Gamboa y Sánchez (1970) aseguran que Brenes era más conocido en el extranjero que en su pueblo natal y en su país. En reconocimiento a su labor, fue propuesto y aceptado como socio de The Torrey Botanic Club, la más antigua sociedad botánica de los Estados Unidos (Monge, 1941).

Vitalía Sáenz, su asistente en el herbario, acotaba que "nuestro botánico nacional, con su prestigio, ha colocado al país en un alto peldaño ante el mundo científico, especialmente por dar a conocer la riqueza florística costarricense" (Sáenz, 1945), a la vez que lo consideraba un eminente científico, reconocía que Brenes mostraba cierto desdén hacia sus propios méritos.

El paso de Brenes por el Herbario Nacional resalta al lado de los investigadores extranjeros que fundaron e incrementaron las colecciones de plantas, tales como el danés Anders S. Oersted (Oersted, 2010), el suizo Adolphe Tonduz (Dauphin, 2017) y el ya citado Pittier, quien con el belga Théophile Durand escribió la gran obra Primitiae Florae Costaricensis (Pittier y Durand, 1898).

Brenes dedicó casi 20 años de su vida a la exploración botánica, que coinciden con el mayor incremento en el conocimiento de la flora costarricense. De hecho, en el libro Flora of Costa Rica (1937), se lee lo siguiente sobre Brenes:

De los costarricenses que han prestado seria atención a la flora nativa de su país y que han sido preeminentes por su trabajo, es el profesor Alberto M. Brenes, botánico del Museo Nacional, por muchos años, quien ha llevado adelante por todo el país, el trabajo de exploración emprendido por Pittier después de que éste abandonó el país. Con inigualable fervor y devoción ha continuado sus colecciones hasta el presente y ha acumulado un herbario de más de 20000 números. Por el volumen y el valor de sus colecciones, no tiene rival en Centroamérica. De hecho, es incuestionable, si alguien lo ha sobrepasado aun en la América del Sur, a no ser por botánicos tales como Spruce Glaziou y Ducke en el Brasil.

\begin{tabular}{|c|c|c|}
\hline 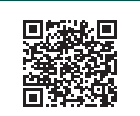 & (c) (i) & 193 \\
\hline
\end{tabular}




\section{Revista de CIENCIAS AMBIENTALES Tropical Journal of Environmental Sciences}

Revista de Ciencias Ambientales (Trop J Environ Sci) e-ISSN: 2215-3896 (Enero-Junio, 2020) . Vol 54(1): 190-199. DOI: https://doi.org/10.15359/rca.54-1.11

Open Access: www.revistas.una.ac.cr/ambientales e-mail: revista.ambientales@una.ac.cr

Salazar H.

El autor de esa obra, uno de los documentos histórico-científicos más importantes, no solo porque actualiza el conocimiento de nuestra vegetación, sino que también por el recuento histórico que hace de la exploración botánica en Costa Rica, fue el célebre experto en plantas, el estadounidense Paul C. Standley. Este inapelable juicio, más el análisis de toda la labor de Brenes, nos permite calificarlo, con todo merecimiento, como el primer botánico costarricense.

\section{Apasionado por las orquídeas y su terruño}

Desde pequeño, Brenes mostró una fuerte predilección por la naturaleza, y en particular por las plantas. Mientras fue profesor o jefe de la Sección de Botánica, cada vez que podía iba a San Ramón, para aumentar sus colecciones. Conoció todo el cantón, palmo a palmo.

Fulgencio González Valverde, su ayudante de campo en San Ramón, recuerda que iban a recolectar plantas a Playa Los Loros (Tárcoles) y a la finca de Nicolás Orlich Zamora en Piedades Sur de San Ramón, entre otros lugares, a caballo o a pie (Ortiz, 1984). González recuerda a Brenes como un hombre muy culto y correcto, aunque de muy pocas amistades. Nunca iba a fiestas, pero era asiduo fumador de cigarrillos que él mismo enrollaba, y a los que añadía un pedazo de algodón, como filtro; además, siempre tenía su traguito de licor en la casa. No olvidaba González que Brenes llamaba la "fulgenciana" a una orquídea que él mismo había recolectado en uno de los tantos viajes que hicieron juntos.

Por su parte, Jiménez (1945) considera a Brenes como el más grande orquideólogo del país, superando en mucho a sus predecesores suizos Pittier y Tonduz. Al respecto, uno de sus principales colegas fue Rudolf Schlechter, del Jardín Botánico de Berlin-Dhalem.

Un trabajo excepcional, en torno a la tipificación de las orquídeas enviadas por Brenes a Schlechter, lo realizó Kerry Barringer, en un documento que, además de aportar datos biográficos, contiene gran cantidad de información sobre los especímenes propiamente dichos (Barringer, 1986). Ahí se percibe que Brenes envió ejemplares en dos períodos: a principios del siglo XX, cuando trabajaba para Pittier, y alrededor de 1922, cuando iniciaba la jefatura de la Sección de Botánica del Museo Nacional. Las colecciones de orquídeas enviadas por Brenes a Schlechter fueron descritas por este en su Beiträge zur Orchideenkunde von Zentralamerika, bajo el título Orchidaceae Brenesianae (Schlechter, 1923).

Lamentablemente, todos los especímenes tipo (holotipos) descritos por Schlechter y depositados en el Museo Botánico de Berlin-Dahlem fueron destruidos el 1. ${ }^{\circ}$ de marzo de 1943, durante la Segunda Guerra Mundial, en un bombardeo que sufrió la ciudad de Berlín. Fue Oakes Ames quien reorganizó parte de la información de Schlechter, utilizando los bocetos, algunos de los cuales fungen como lectotipos (Barringer, 1986).

De la colección de Brenes, Schlechter (1923) consideró 146 especies como nuevas para la ciencia, de las cuales el 83 \% procedían del cantón de San Ramón, y de estas el 53 \% fueron recolectadas en la localidad de San Pedro. Por esta razón, y con mucho entusiasmo, Schlechter calificó a San Ramón como "El Dorado" de las orquídeas. Conviene aclarar que la tipificación hecha por Barringer (1986) es de apenas 93 especies, es decir, del $63 \%$ del total.

\begin{tabular}{|c|c|c|}
\hline 品的至 & (c) & 194 \\
\hline
\end{tabular}




\section{Revista de CIENCIAS AMBIENTALES Tropical Journal of Environmental Sciences}

Revista de Ciencias Ambientales (Trop J Environ Sci) e-ISSN: 2215-3896 (Enero-Junio, 2020) . Vol 54(1): 190-199. DOI: https://doi.org/10.15359/rca.54-1.11

Open Access: www.revistas.una.ac.cr/ambientales e-mail: revista.ambientales@una.ac.cr

Salazar H.

Para concluir esta sección, es oportuno señalar que se conocen como brenesias las especies que fueron bautizadas utilizando el apellido Brenes, cuya forma latinizada es brenesii. En cuanto a las albertias, también denominadas en honor a él, son menos conocidas en razón de que es más frecuente utilizar el apellido y no el nombre para honrar a una persona con un nombre científico.

Solamente de la familia Orchidaceae (orquídeas), Schlechter denominó 17 especies nuevas y un nuevo género (Brenesia costaricensis), así como cuatro especies de albertias (Barringer, 1986). El entusiasmo de Schlechter con las orquídeas costarricenses y con la colección de Brenes fue tal, que denominó brenesias en casi todos los géneros de orquídeas con las que trabajó. Las albertias siempre aparecen en géneros en los cuales ya había denominado una brenesia, como por ejemplo Elleanthus, Epidendrum, Maxillaria y Stelis.

Schlechter también denominó varias especies de orquídeas recolectadas por Brenes como costaricensis (Barringer, 1986), con lo cual se comenzó a poner en alto el nombre de Costa Rica $y$, sin duda, fueron esas primeras denominaciones de especies costarricenses las que contribuyeron a poner a este pequeño país a la vanguardia del conocimiento de la diversidad biológica internacional.

\section{El micólogo}

Brenes fue un entusiasta recolector de hongos. De hecho, su colección, depositada también en el Museo Botánico de Berlín-Dahlem (Hiepko, 1987), fue objeto de varios análisis, especialmente, por parte de los especialistas Hans Sydow y Franz Petrak, bajo el título Fungi costaricenses a cl. Prof. Alberto M. Brenes Collecti. En estas publicaciones se identifican 141 especies, de las cuales ocho están dedicadas a Costa Rica, 10 a Brenes, y un nuevo género dedicado a él (Sydow y Petrak, 1929; 1937).

Las actividades de Brenes como micólogo son escasamente documentadas en Costa Rica, probablemente porque las publicaciones se hicieron en idioma alemán, pero no por ello dejan de ser importantes. De hecho, el reino fungi es uno de los menos estudiados, en especial aquellos grupos que no son macrohongos. En Costa Rica, los esfuerzos de recolección que hiciera Brenes de este grupo podrían considerarse como pioneros, pues fue gracias a sus adecuadas técnicas de recolección y preservación que se facilitó la identificación de las diferentes especies por parte de los especialistas alemanes, además del aporte en relación con las plantas hospedantes.

En los archivos del Departamento de Protección de Patrimonio Histórico del Museo Nacional se encuentra una lista de al menos 44 muestras de macrohongos de la colección de Brenes. Algunas de ellas están identificadas en el nivel de especie, y otras en el nivel de género por John Albert Stevenson, del Departamento de Agricultura de los Estados Unidos.

\section{El excursionista}

Brenes fue un excursionista incansable por cuenta propia, además de que participó en varias giras, acompañando a especialistas extranjeros. Una de las actividades más importantes en las

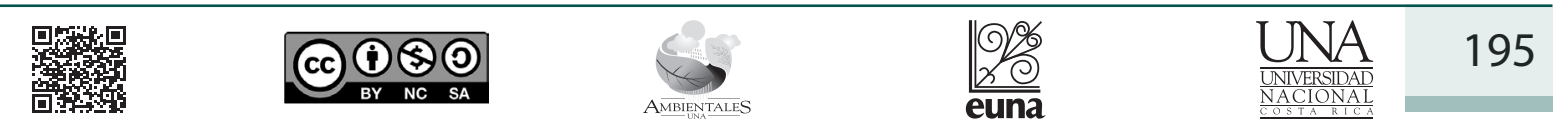




\section{Revista de CIENCIAS AMBIENTALES Tropical Journal of Environmental Sciences}

Revista de Ciencias Ambientales (Trop J Environ Sci) e-ISSN: 2215-3896 (Enero-Junio, 2020) . Vol 54(1): 190-199. DOI: https://doi.org/10.15359/rca.54-1.11

Open Access: www.revistas.una.ac.cr/ambientales e-mail: revista.ambientales@una.ac.cr

Salazar H.

que participó fue con la Comisión Científica Austriaca, liderada por Otto Porsch. El principal propósito de los austriacos era hacer investigaciones botánicas y zoológicas, acopiar material biológico y minerales, así como realizar registros fotográficos para exhibirlos en el Museo de Historia Natural de Viena. Uno de los objetivos específicos era estudiar las relaciones ecológicas entre las aves y las plantas en el proceso de polinización. La escogencia de Costa Rica para esta expedición ocurrió porque ya existían suficientes registros de la biodiversidad costarricense, y Porsch conocía muy bien esa información.

Afortunadamente, también encontraron en Brenes - ya con poco más de sesenta años de edad- el guía perfecto para llevarlos por gran parte del territorio nacional, en una actividad científica que se convirtió en una especie de frenesí para los europeos, que deseaban recolectar tantos especímenes como les fuera posible y conocer los nombres de plantas y animales, así como las relaciones ecológicas entre ellas. Y contaban con Brenes, para dar respuesta a cuantas preguntas le hacían.

Ahí estuvo él, mano a mano con Otto Porsch, apasionado por los colibríes; Eduard Reimoser, aracnólogo y entomólogo; Moritz Sassi, ornitólogo y director del Museo de Historia Natural de Austria; Otto Koller, ictiólogo y mastozoólogo; Georg Cufodontis, botánico; Rudolph Zimara, guía de la expedición y herpetólogo; W. Möller, ornitólogo; Alfred Stadler, taxidermista; y F. Jarkowsky, fotógrafo.

Además, fue guía del alemán Walter Kupper, especialista en musgos y líquenes, grupo botánico del que Brenes tenía algún conocimiento, pues en su juventud había hecho algunas recolecciones de briófitos; así como también de Hans Sydow, otro alemán, quien era experto en hongos (Sáenz, 1945). Tiempo después, aunque ya de edad más avanzada y con menos andanzas en el campo, fue compañía del célebre botánico Paul C. Standley.

\section{Acerca de sus publicaciones}

Se conoce apenas un trabajo escrito por Brenes, con el título Con la Comisión Científica Austriaca en el Guanacaste, pero corresponde al informe de una gira de campo; no fue él quien lo publicó, sino el botánico Alfonso Jiménez Muñoz, curador del Herbario del Museo Nacional (Jiménez, 1956). El hecho de que este indicara que se trataba de la única publicación de Brenes, quizás desestimuló la investigación relacionada con su obra, al menos de la escrita. Aunque es cierto que él no hizo publicaciones formales, ello no significa que no escribiera nada. Muy por el contrario, al parecer lo hacía cada vez que podía. Al fin de cuentas, como buen ramonense, tenía en su sangre pluma de escritor.

Lo hacía en trozos de papel nuevo o de desecho, e incluso en las mismas cajetillas de cigarros que desarmaba para escribir por detrás. Por supuesto que ahí no se plasmaban poemas, sino notas científicas que debía recordar. No cabe la menor duda de que muchas de ellas solo fueron para él, ya que la letra es sumamente pequeña y de muy difícil interpretación, pero otras tenían toda la intención de ser, si no publicadas, al menos compartidas con otros.

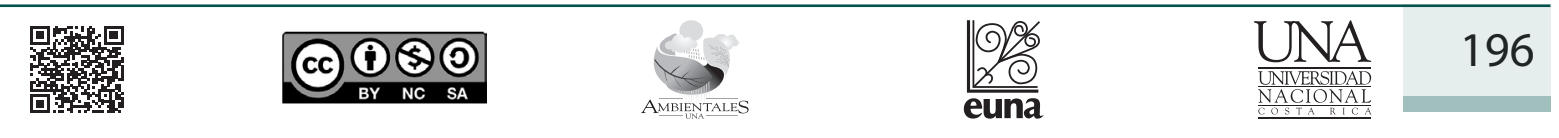




\section{Revista de CIENCIAS AMBIENTALES Tropical Journal of Environmental Sciences}

Revista de Ciencias Ambientales (Trop J Environ Sci) e-ISSN: 2215-3896 (Enero-Junio, 2020) . Vol 54(1): 190-199. DOI: https://doi.org/10.15359/rca.54-1.11

Open Access: www.revistas.una.ac.cr/ambientales e-mail: revista.ambientales@una.ac.cr

Cabe destacar que, en la labor de rescate de su obra escrita, Salazar (2009) pudo recopilar cuatro de sus textos, correspondientes a los informes con la Comisión Austriaca en Guanacaste y con la Comisión Científica Austriaca en Golfo Dulce, así como la lista de Orquídeas de Costa Rica y Notas orquideológicas, que es una especie de bitácora en la que anotaba, con fecha, la floración y los detalles de su colección privada de orquídeas, algunas de las cuales tienen dibujos hechos a lápiz (Figura 2).

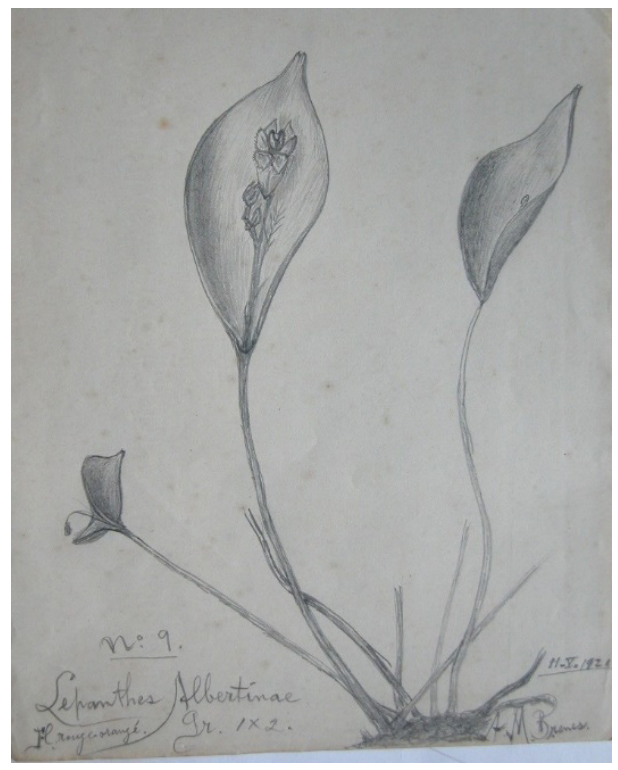

Figura 2. Dibujo a lápiz de la orquídea Lepanthes Albertinae, que data de 1921. Figure 2. Pencil drawing of the Lepanthes Albertinae orchid, dating from 1921.

\section{Epílogo}

Brenes fue siempre un hombre solitario, incomprendido quizás, y abandonado incluso por sus mismos coterráneos al final de sus días, que los soportó en medio de una gran pobreza.

Aunque casi no publicó nada, fue muy generoso en notas sobre cada uno de los especímenes botánicos recolectados, referentes a los caracteres taxonómicos, que son de gran ayuda para la identificación. Como lo hizo notar el propio Schlechter, Brenes fue el primero en dar nota exacta de los colores de las flores de las orquídeas vivas, detalle que los recolectores por lo general olvidan, pues las flores delicadas de estas plantas pierden toda traza de su notable colorido al ser secadas para herbario.

Asimismo, por su labor tan entusiasta en el campo de la orquideología y la micología, así como en la recolección sistemática de especímenes de estos grupos, debería reconocérsele como el precursor de estas ciencias en Costa Rica.

\begin{tabular}{|c|c|c|}
\hline 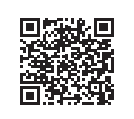 & (c) (i) (9) (D) & 197 \\
\hline
\end{tabular}




\section{Revista de CIENCIAS AMBIENTALES Tropical Journal of Environmental Sciences}

Revista de Ciencias Ambientales (Trop J Environ Sci) e-ISSN: 2215-3896 (Enero-Junio, 2020) . Vol 54(1): 190-199. DOI: https://doi.org/10.15359/rca.54-1.11

Open Access: www.revistas.una.ac.cr/ambientales e-mail: revista.ambientales@una.ac.cr

Salazar H.

El conocimiento de la biodiversidad costarricense, y en particular de su flora, así como la existencia de numerosos epítetos de nombres científicos como costaricensis y ramonensis se deben, sin lugar a dudas, a las recolecciones hechas por él. Fue así como Costa Rica y San Ramón se ganaron un lugar en los mapas del mundo, para los estudiosos de la naturaleza. Sin la figura de Brenes, desde el punto de vista del conocimiento de la biodiversidad, seguramente, este pueblo hubiese sido un lugar similar a cualquier otro. No obstante, los recorridos de este ilustre ramonense por su cantón natal, haciendo recolecciones sistemáticas de cuanto pudiera, hicieron esa gran diferencia. Como un tributo a su memoria, la Reserva Biológica que lleva su nombre, localizada también en San Ramón, sigue manteniendo vivo su espíritu, y desde ahí se continúan aportando nuevos conocimientos a la ciencia costarricense y mundial.

Para concluir, debemos decir que hay personas que nunca mueren. Y este es el caso de Alberto Manuel Brenes, quien, con su labor silenciosa, pero fecunda supo dejar una huella profunda e imperecedera en su paso por esta Tierra, logró alcanzar la gloria de la inmortalidad y a la vez encontrar el camino hacia las estrellas.

\section{0. Ética y conflicto de intereses}

La persona autora declara que ha cumplido totalmente con todos los requisitos éticos y legales pertinentes en la producción del manuscrito; que no hay conflictos de intereses de ningún tipo; y que está totalmente de acuerdo con la versión final editada del artículo.

\section{Referencias}

Barringer, K. (1986). Typification of Schlechter's Costa Rican Orchidaceae. I Types Collected by A. Brenes. Fieldiana, Botany, 17: 1-24. https://doi.org/10.5962/bhl.title.2664

Cruz, O. (1990). Científico costarricense Alberto Manuel Brenes Mora (biografía 1870-1948). 120 aniversario del natalicio del Dr. Alberto Ml. Brenes Mora. Asociación Ramonense para la Conservación del Ambiente (A. R. C. A), San Ramón, Costa Rica. Mimeografiado. 2 p.

Dauphin, G. (2017). Adolphe Tonduz y la época de oro de la botánica en Costa Rica. Cartago. Editorial Tecnológica de Costa Rica.

Echavarría, T. (1944). Alberto M. Brenes. Apuntes sobre su vida. I. G. B. N. 7882 . Departamento de Protección del Patrimonio Histórico. Museo Nacional de Costa Rica. 6 p.

Hiepko, P. (1987). The collections of the Botanical Museum Berlin-Dahlem (B) and their history. Englera, 7: 219-252.

Jiménez, J. A. (1945). Un costarricense ilustre. Revista del Instituto de Defensa del Café de Costa Rica, XV (131): 523-525.

\begin{tabular}{|c|c|c|}
\hline 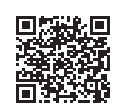 & (c) (1) () & 198 \\
\hline
\end{tabular}




\section{Revista de CIENCIAS AMBIENTALES Tropical Journal of Environmental Sciences}

Revista de Ciencias Ambientales (Trop J Environ Sci) e-ISSN: 2215-3896 (Enero-Junio, 2020) . Vol 54(1): 190-199. DOI: https://doi.org/10.15359/rca.54-1.11

Open Access: www.revistas.una.ac.cr/ambientales e-mail: revista.ambientales@una.ac.cr

Salazar H.

La Gaceta. (1970). Declárese benemérito de la ciencia costarricense al botánico prof.: Alberto Manuel Brenes Mora. La Gaceta, N.ำ183, 19 de agosto de 1970.

Monge, F. (1941). Valores costarricenses: biografía de don Alberto María Brenes. La Prensa Libre, 26 de junio de 1941, p. 12.

Oersted, A. S. (2010). La América Central. San José, Costa Rica. Editorial Universidad de Costa Rica.

Ortiz, F. (1984). Perfil de don Alberto Brenes Mora. Seminario B-0400: Historia de la Biología en Costa Rica (II-Ciclo). Universidad de Costa Rica (mimeografiado). 10 p.

Pittier, H. y T. H. Durand. (1898). Primitiae Florae Costaricensis. Instituto Físico Geográfico Nacional, Costa Rica.

Sáenz, V. (1945). Prof. don Alberto M. Brenes “Nuestro Botánico Nacional”. Boletín del Museo Nacional. 1 (2): 7-9.

Salas, O. (1949). Alberto Ml. Brenes. Carpeta N. ${ }^{\circ}$ 7882. Departamento de Protección del Patrimonio Histórico. Museo Nacional de Costa Rica. 4 p.

Salazar, A. H. (2009). Alberto Ml. Brenes: el naturalista. San José, Editorial Universidad de Costa Rica.

Schlechter, R. (1923). Beiträge zur Orchideenkunde von Zentralamerika. II. Additamenta ad Orchideologiam Costaricensem. Repertorium specierum novarum regni vegetabilis XIX: 158-269.

Standley, P. C. (1937). Flora of Costa Rica. Part I. Chicago, Field Museum of Natural History, Botanical Series. Vol. XVIII. 780 p.

Sydow, H., Petrak, F. (1929). Fungi costaricenses a cl. Prof. Alberto M. Brenes collecti. Series prima. Annales Mycologici XXVII (1/2): 1-86.

Sydow, H., Petrak, F. (1937). Fungi costaricenses a cl. Prof. Alberto M. Brenes collecti. Series Secunda. Annales Mycologici. XXXV (2): 6-97.

Valerio, J. (1940). Informe rendido a la Secretaría de Educación Pública sobre la labor realizada en 1939. Museo Nacional. Serie Historia del Museo, vol. I, parte II. P. 4-8.

\begin{tabular}{|c|c|c|}
\hline 唃要 & (c) (i) & 199 \\
\hline
\end{tabular}

